\title{
Electrochemical Study on A Heme-Histidine-Sodium Decyl Sulfate Nano Micelles Complex Artificial Peroxidase
}

\author{
Di LI \\ School of Life Sciences \\ Henan University \\ Kaifeng, China \\ e-mail: 1742043028@qq.com \\ Fangyong GENG \\ School of Life Sciences \\ Henan University \\ Kaifeng, China \\ e-mail: 1742043028@qq.com
}

\section{Jun HONG*}

School of Life Sciences and Institute of Biotechnology

Henan University

Kaifeng, China

e-mail: hongjun@henu.edu.cn

\author{
Yushuai ZHANG \\ School of Life Sciences \\ Henan University \\ Kaifeng, China \\ e-mail: 1742043028@qq.com
}

\author{
Baolin XIAO \\ School of Life Sciences \\ Henan University \\ Kaifeng, China \\ e-mail: 78448742@qq.com
}

\author{
Tao HONG* \\ School of Art \\ Henan University \\ Kaifeng, China \\ e-mail: hhyt226@163.com
}

Di Li, Yushuai Zhang and Fangyong Geng contributed equally to this work.

\begin{abstract}
A heme-histidine-sodium decyl sulfate nano micelles complex with peroxidase activity (MAP) was constructed self-assembly and immobilized on a hydroxy fullerene modified glassy carbon electrode. The cyclic voltammograms of the modified electrode with a pair of redox peaks were investigated. The formal potential of the MAP modified electrode was determined to be $-0.312 \mathrm{~V}$, at a scan rate of $0.05 \mathrm{~V} / \mathrm{s}$ in $50 \mathrm{mmol} / \mathrm{L}$ sodium phosphate buffer solution (pH 8.0). The electron transfer rate constant $\left(k_{s}\right)$ and cathodic transfer coefficient $(\alpha)$ were $1.96 \mathrm{~s}^{-1}$ and 0.4 , respectively. The cathodic peak current of the MAP modified electrode responses linearly to hydrogen peroxide ranging from $300 \mu \mathrm{mol} / \mathrm{L}$ to $1700 \mu \mathrm{mol} / \mathrm{L}$. The detection limit was concluded to be $32 \mu \mathrm{mol} / \mathrm{L}$. The apparent Michaelis-Menten constant $\left(\mathrm{K}_{\mathrm{m}}{ }^{\mathrm{app}}\right)$ could be determined to be $1.08 \mathrm{mmol} / \mathrm{L}$.
\end{abstract}

Keywords-heme; nano micelle; artificial peroxidase; electrochemistry

\section{INTRODUCTION}

It is an important aspect in the design of a heme protein through electrochemical investigation and simulation the functional or structural analogues. The electrochemical study of a heme protein and its mimic enzyme may reveal the electron transfer reaction principle and the energy conversion process of the heme protein. Moreover, it could provide theories foundation for the new type biosensors developments [1-2].

Heme is an important cofactor of a heme protein (horseradish peroxidase, cytochrome c, etc.) and the catalytic activity center of a heme-containing enzyme. Sodium decyl sulfate, which has a hydrophobic tail and hydrophilic head, may form stable nano micelles in aqueous solution when its concentration is above CMC concentration. Because of the shorter chain length, the formed sodium decyl sulfate micelles have smaller diameter, stronger hydrophobic interaction and electrostatic effect [3-6], which may provide a hydrophobic environment and enhance the stability and activity of an artificial peroxidase [7-10].

Nano materials possess many excellent characters, such as large specific surface area, good conductivity, and biocompatibility, so it has an important role in electrochemical research and a wide range of applications. Hydroxy fullerenes are a product of functionalized fullerene, which is added with hydroxyl group on the surface of fullerene to increase its solubility in water and enhance the biocompatibility [11-12].

Chitosan is a linear polysaccharide, where $\beta$-(1-4)-linked D-glucosamine and N-acetyl-D-glucosamine are distributed stochastically. Its monomer molecular formula is C6H11NO4. Chitosan has been used in immobilization technology, including immobilize enzyme on an electrode surface for its advantages of good biocompatibility, strong stability and simple structure [13].

In the present study, heme-histidine-sodium decyl sulfate nano micelles complex artificial peroxidase (MAP) was constructed self-assembly and immobilized on a glassy carbon electrode (GCE). Then the electrode was covered with a chitosan membrane. The MAP modified electrode shows a good response to hydrogen peroxide concentration. 


\section{EXPERIMENTAL}

\section{A. Reagents}

Heme and sodium decyl sulfate (SDeS) are obtained from Sigma (Saint Louis, MO, USA). Hydroxy Fullerenes (HFs) is bought from Shenzhen Nanotech Port Ltd. Co. (China). Sodium dihydrogen phosphate, disodium hydrogen phosphate, chitosan (Chi) and hydrogen peroxide are purchased from Shanghai Chemicals Company, China. All chemicals used in these investigations are of analytical grade.

\section{B. Artificial Peroxidase Preparation}

Typical heme-histidine-SDeS nano micelles complex artificial peroxidase (MAP) was constructed by mixing heme, histidine (His) and SDeS in 50mmol/L, pH8.0 PBS to form a homogeneous colloidal solution [14-17]. The final concentrations of heme, SDeS and His were 2, 50 and $50 \mathrm{mmol} / \mathrm{L}$, respectively. The size of MAP nano micelle was 3.45nm, which was determined using a Zeta Plus Dynamic light scattering (DLS) instrument, Brookhaven Instruments Corporation, America. The chemical structures, preparation process and size of MAP nano-micelle could be seen in Scheme 1.
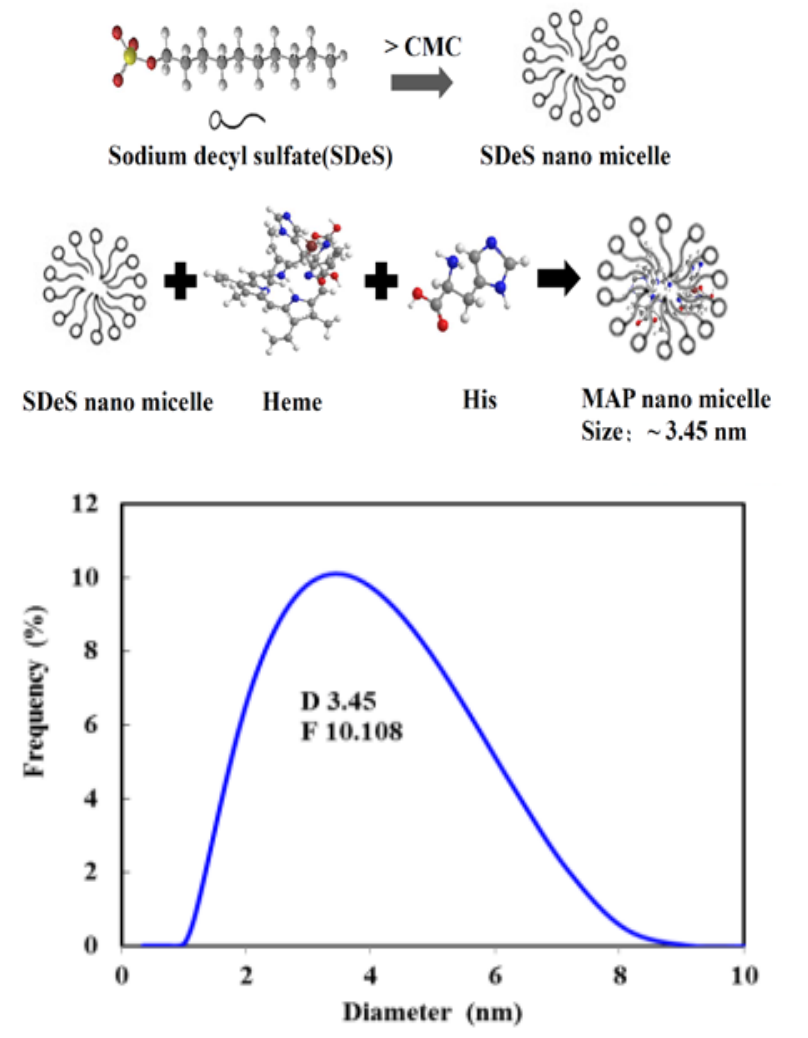

Scheme 1 Preparation process and size of MAP nano-micelle

\section{Fabrication of Chi/MAP/Hfs/GCE}

At first, a GCE was prepared similar to the procedure given in previous literature [18-19]. Then, $3 \mu \mathrm{L}$ HFs ( $2 \mathrm{mg} / \mathrm{mL}$ ) was dropped on the prepared GCE. After drying for $4 \mathrm{~h}$ at room temperature, $4 \mu \mathrm{L}$ MAP was dropped on the electrode and dried at $25^{\circ} \mathrm{C}$. Finally, $4 \mu \mathrm{l}$ Chi $(0.5 \%)$ was covered on the surface of the electrode and dried at $25^{\circ} \mathrm{C}$.

\section{RESULTS AND DISCUSSION}

\section{A. Electrochemical Properties of Chi/MAP/Hfs/GCE}

The cyclic voltammograms (CVs) of (a)bare GCE, (b)Chi/HFs/GCE, (c)Chi/His/HFs/GCE, (d)Chi/SDeS/HFs/GCE, (e)Chi/heme/HFs/GCE and (f)Chi/MAP/HFs/GCE were shown in Fig. 1A.

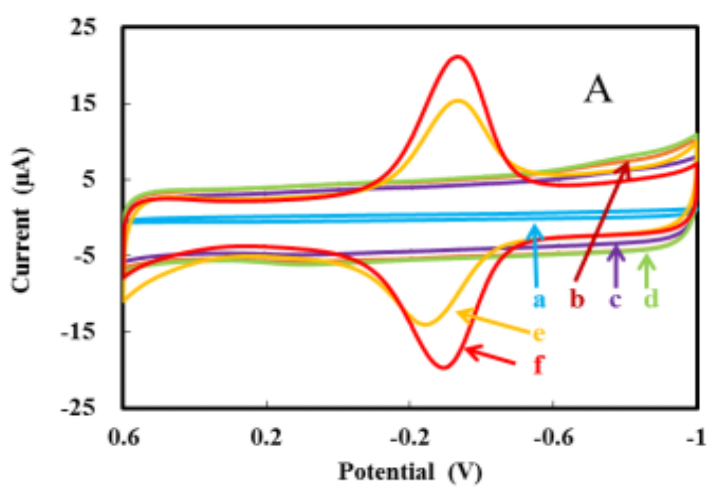

Figure 1A. The cyclic voltammograms (CVs) of (a) bare GCE, (b)

Chi/HFs/GCE, (c) Chi/His/HFs/GCE, (d) Chi/SDeS/HFs/GCE, (e) $\mathrm{Chi} / \mathrm{Heme} / \mathrm{HFs} / \mathrm{GCE}$ and (f) $\mathrm{Chi} / \mathrm{MAP} / \mathrm{HFs} / \mathrm{GCE}$. The tests were done in $50 \mathrm{mmol} / \mathrm{L}$, pH8.0 PBS at a scan rate of $0.05 \mathrm{Vs}^{-1}$

It could be seen that curve (f) shows a pair of stronger redox peaks respect to that of curve (e). The ratio of cathodic (Ipc) to anodic peak current (Ipa) was calculated to be 0.9. The difference between anodic (Epa) and cathodic peak potential (Epc) was determined to be $0.04 \mathrm{~V}$. Thus, the electrochemical process of Chi/MAP/HFs/GCE (f) was quasi-reversible [20]. Moreover, the formal potential $\left(\mathrm{E}^{\mathrm{o}}=\mathrm{Epa} / 2+\mathrm{Epc} / 2\right)$ was determined to be $-0.312 \mathrm{~V}$ versus $\mathrm{Ag} / \mathrm{AgCl}$. In the meanwhile, no redox peaks could be seen in curve (a), curve (b), curve (c) and curve (d).

The CVs of the Chi/MAP/HFs/GCE in 50mmol/L PBS at different $\mathrm{pH}$ values were illustrated in Fig. $2 \mathrm{~B}$.

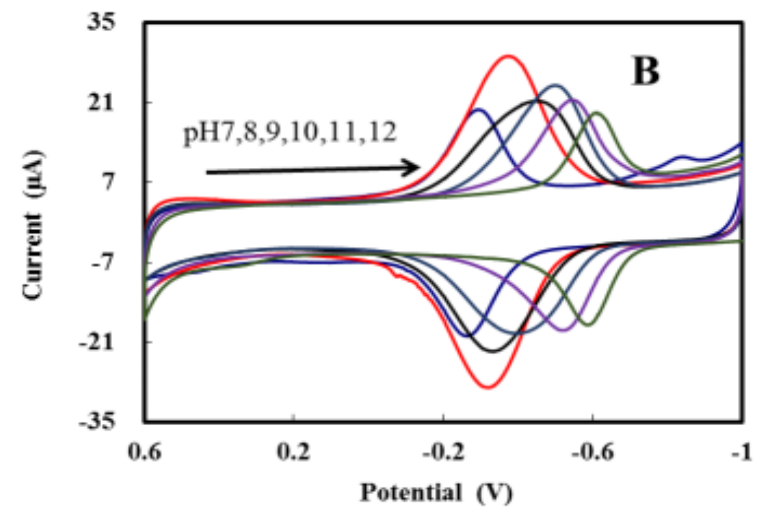

Figure 2B. CVs of Chi/MAP/HFs/GCE in 50mmol/L PBS at different $\mathrm{pH}$ values (from left to right): 7, 8, 9, 10,11 and 12, respectively

The maximum cathodic current was obtained at $\mathrm{pH}$ 8.0, and so, this optimal $\mathrm{pH}$ value was chosen for the next set of 
tests. The formal potential of the MAP modified GCE was $\mathrm{pH}$ dependent for the $\mathrm{pH}$ value ranging from 7 to 12 . The slope $(0.064 \mathrm{~V} / \mathrm{pH}$, Fig. $3 \mathrm{C})$ was close to the Nernst's value $(0.059 \mathrm{~V} / \mathrm{pH})$ for one-electron and one-proton process [21].

Based on the observed CVs of the Chi/MAP/HFs/GCE(Fig. 4D), the peak currents increased with increasing scan rate (v) and were proportional to $v$ in the range of $0.01-1.2 \mathrm{~V} / \mathrm{s}$ (Fig. 5E), indicating that the MAP was stably immobilized on the GCE. The cathodic peak potential altered linearly respect to lnv for the scan rates ranging from 0.5 to $1.0 \mathrm{~V} / \mathrm{s}$ (Fig. $6 \mathrm{~F}$ ).

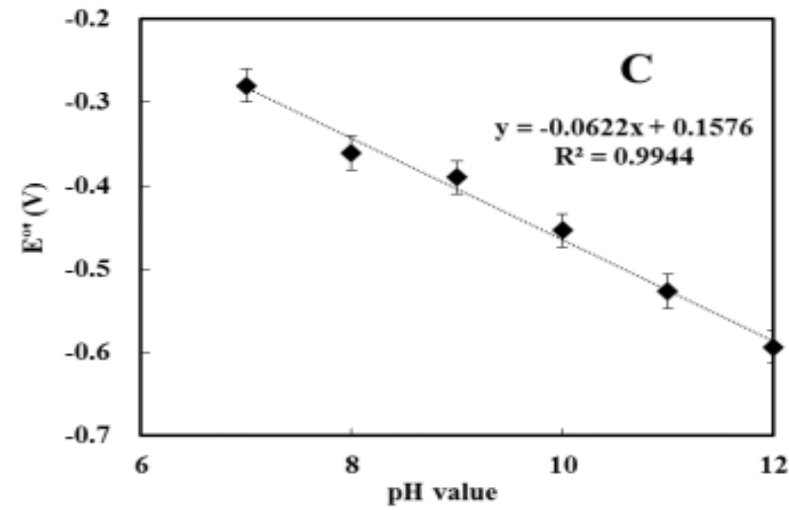

Figure 3C. Relationship between formal potential $\left(\mathrm{E}^{\mathrm{o}}\right)$ and $\mathrm{pH}$ value.

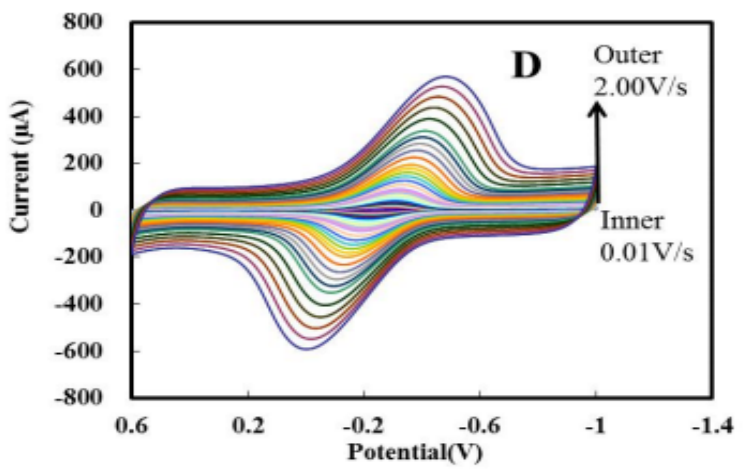

Figure 4D. CVs of the Chi/MAP/HFs/GCE in 50mmol/L, pH $8.0 \mathrm{PBS}$ at the different scan rates (from inner to outer): $0.01,0.02,0.03 \ldots 1.2 \mathrm{~V} / \mathrm{s}$, respectively.

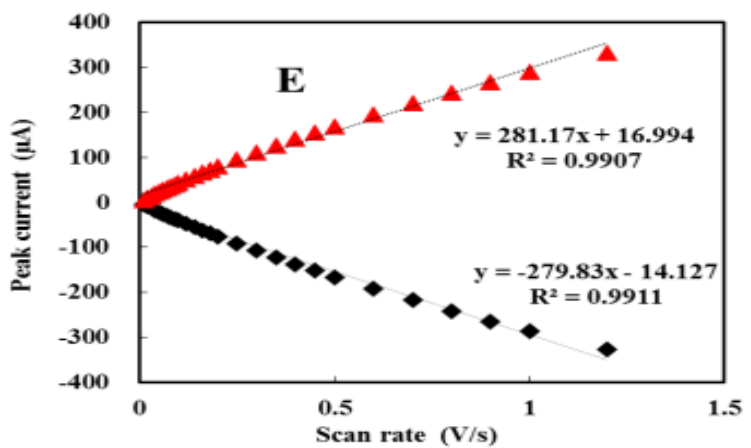

Figure 5E. Relationship between peak current $\left(I_{p}\right)$ and scan rate (v).

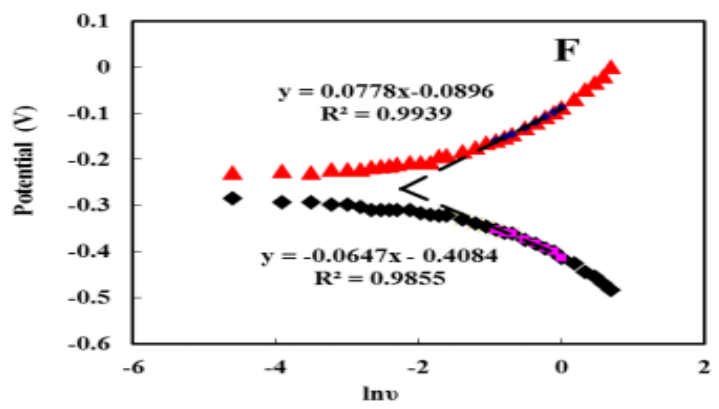

Figure 6F. Relationship between peak potential $\left(\mathrm{E}_{\mathrm{p}}\right)$ and $\ln$.

Then the number of electron (n), cathodic electron transfer coefficient $(\alpha)$, apparent heterogeneous electron transfer rate constant $\left(\mathrm{k}_{\mathrm{s}}\right)$ and the average surface concentration of electroactive materials (MAP) on the surface of GCE $(\Gamma)$ could be concluded to be $1,0.4,1.96 \mathrm{~s}^{-1}$ and $9.10 \times 10^{-9} \mathrm{~mol} / \mathrm{cm}^{2}$, respectively [19-23]. Moreover, the size of MAP nano micelle was $3.45 \mathrm{~nm}$ (Scheme 1), then the theoretical concentration of one layer MAP could be calculated to be $1.40 \times 10^{-11} \mathrm{~mol} / \mathrm{cm}^{2}$ [12]. So the distribution of MAP on the surface of the modified electrode was very concentrated.

\section{B. Electro-Catalytic Properties of Chi/MAP/Hfs/GCE}

The linear sweep voltammograms (LSVs) of Chi/MAP/HFs/GCE and Chi/Heme/HFs/GCE were presented in Fig. 7A and Fig. 8B.

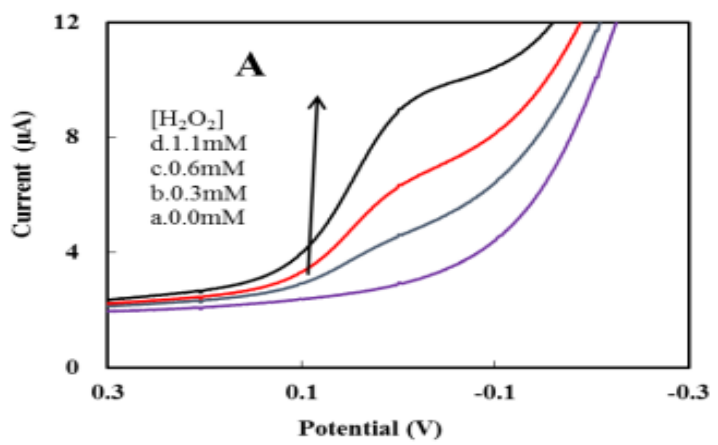

Figure 7A. The linear sweep voltammograms (LSVs) of Chi/MAP/HFs/GCE.

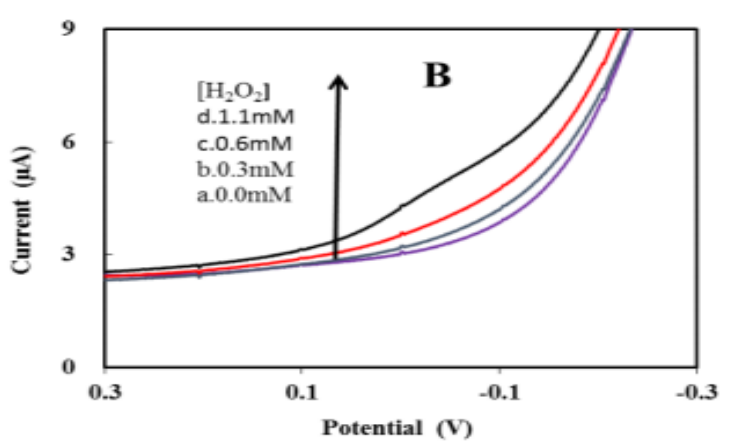

Figure 8B. Chi/heme/HFs/GCE in the absence and presence of 0.3, 0.6 and $1.1 \mathrm{mmol} / \mathrm{L} \mathrm{H}_{2} \mathrm{O}_{2}$ in $50 \mathrm{mmol} / \mathrm{L}$ PBS (pH8.0), at a scan rate of $0.05 \mathrm{Vs}^{-1}$ 
It could be seen that the MAP modified electrode show better response to $\mathrm{H}_{2} \mathrm{O}_{2}$, respect to that of Chi/heme/HFs/GCE. In the meanwhile, either Chi/SDeS/HFs/GCE or Chi/His/HFs/GCE shows almost no response to $\mathrm{H}_{2} \mathrm{O}_{2}$ (data not shown). It seems that His and SDeS play important roles for the structure of MAP, and the heme acts as an essential role for the catalytical function of MAP. The MAP modified electrode shows more efficient catalytic activity respect to that of heme.

The possible electro-catalytic mechanism on Chi/MAP/HFs/GCE reaction could be expressed as the following [18]:

$$
\operatorname{MAP}\left[\operatorname{Heme}\left(\mathrm{Fe}^{\mathrm{II}}-\mathrm{H}\right)\right]+1 / 2 \mathrm{H}_{2} \mathrm{O}_{2}=\operatorname{MAP}\left[\operatorname{Heme}\left(\mathrm{Fe}^{\mathrm{III}}\right)\right]+\mathrm{H}_{2} \mathrm{O}
$$

Fig. 9C shows the relationship between peak current (Ip) and $\mathrm{H}_{2} \mathrm{O}_{2}$ concentration. The linear range was from 300 to $1700 \mu \mathrm{mol} / \mathrm{L}$.

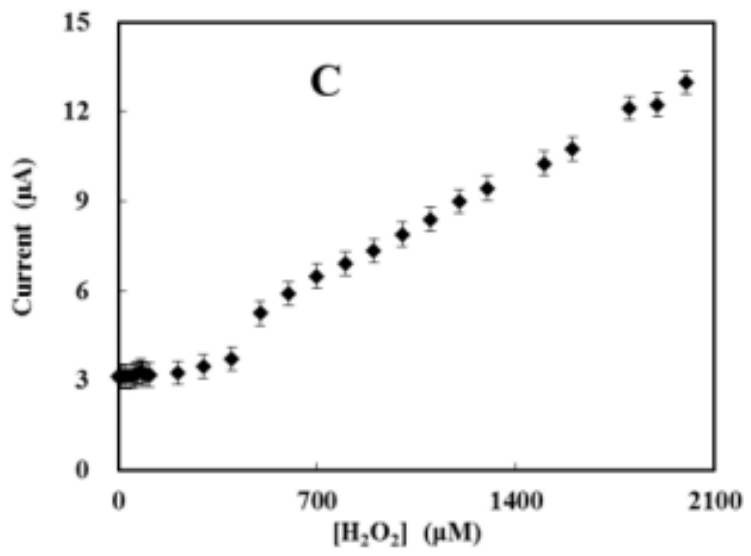

Figure 9C. The relationship between the peak current $\left(\mathrm{I}_{\mathrm{p}}\right)$ and the concentration of $\mathrm{H}_{2} \mathrm{O}_{2}$ (with the typical linear range from 300 to 1700 $\mu \mathrm{mol} / \mathrm{L})$.

The detection limit was $32 \mu \mathrm{mol} / \mathrm{L}$. Then, according to the electrochemical version of the Linewearver-Burk Equation [19-24] and Fig. 10D,

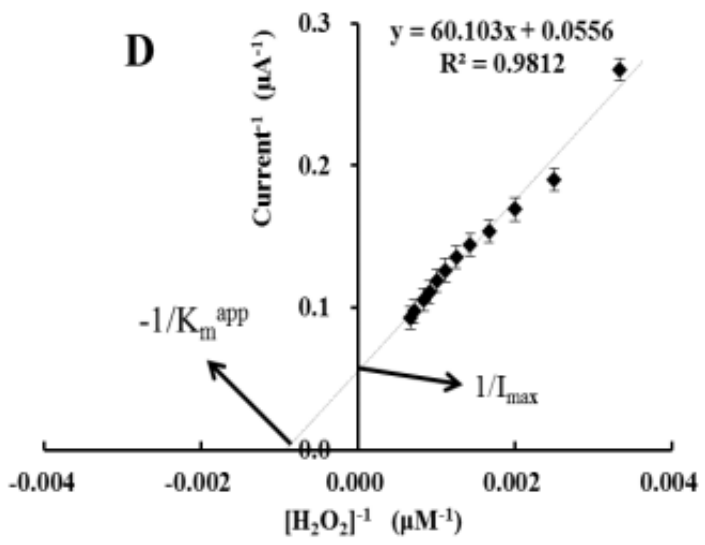

Figure 10D. Lineweaver-Burk plot of MAP.

the apparent Michaelis-Menten constant $\left(\mathrm{K}_{\mathrm{m}}{ }^{\mathrm{app}}\right)$ could be calculated to be $1.08 \mathrm{mmol} / \mathrm{L}$. This value is less than that of HRP/thionine/GCE (1.6mmol/L) [25], HRP/NF/MG/GCE
(2.1mmol/L) [26]. Though it is larger than that of HRP/gelatin/RTILs/GCE $(0.0684 \mathrm{mmol} / \mathrm{L})$ [27], HRP/Au colloid/Cys/glutaraldehyde/Cys/GCE $(0.094 \mathrm{mmol} / \mathrm{L})$ [28], as an electrochemical system without protein, it may have important research significance and potential application value.

\section{CONCLUSIONS}

A heme-histidine-sodium decyl sulfate nano micelles artificial peroxidase (MAP) was constructed and immobilized on a hydroxy fullerene modified glassy carbon electrode. The prepared MAP modified glassy carbon electrode could be used as a biosensor for $\mathrm{H}_{2} \mathrm{O}_{2}$ determination with low detection limit and high sensitivity. MAP could be used instead of native horseradish peroxidase to construct a sensitive $\mathrm{H}_{2} \mathrm{O}_{2}$ biosensor without any protein.

\section{ACKNOWLEDGMENT}

The support of Development Projects of Henan Province Science and Technology (142300410124), Henan University Science Foundation (Y1425013), the Research Council of University of Tehran and Iran National Science Foundation (INSF) are gratefully acknowledged.

\section{REFERENCES}

[1] J. Hirst, A. Sucheta, B. A. C. Ackrell, F. and A. Armstrong, Electrocatalytic voltammetry of succinate dehydrogenase: direct quantification of the catalytic properties of a complex electron-transport enzyme, Journal of the American Chemical Society, vol. 118, pp. 5031-5038, May 1996. (references)

[2] H. A. Heering, J Hirst, and F. A. Armstrong, Interpreting the catalytic voltammetry of electroactive enzymes adsorbed on electrodes, The Journal of Physical Chemistry B, 102, vol. 102, pp. 6889-6902, August 1998.

[3] M. J. Rosen and J. T. Kunjappu, Surfactants and interfacial phenomena, John Wiley \& Sons, 2012..

[4] W. Parker and Song P-S, Protein structures in SDS micelle-protein complexes, Biophys J , vol. 61, pp. 1435-1439, June 1992.

[5] R. Montserret, M. J. Mcleish, A. Böckmann, A. C. Geourjon, and F. Penin, Involvement of electrostatic interactions in the mechanism of peptide folding induced by sodium dodecyl sulfate binding, Biochemistry, vol. 39, pp. 8362-8373, May 2000.

[6] A. A. Moosavi-Movahedi, J. Chamani, M. Gharanfoli, and G. H. Hakimelahi, Differential scanning calorimetric study of the molten globule state of cytochrome c induced by sodium n-dodecyl sulfate, Thermochim Acta, vol. 409, pp. 137-144, January 2004.

[7] M. Z. Atassi and T. Manshouri, Design of peptide enzymes (pepzymes): surface-simulation synthetic peptides that mimic the chymotrypsin and trypsin active sites exhibit the activity and specificity of the respective enzyme, Proceedings of the National Academy of Sciences, vol. 90, pp. 8282-8286, September 1993.

[8] D. F. Tai and W. C. Liaw, Thiolsubtilisin acts as an acetyltransferase in organic solvents, FEBS Lett, vol. 517, pp. 24-26, April 2002.

[9] A. Tramontano, K. D. Janda, and R. A. Lerner, Catalytic antibodies, Science, vol. 234, pp. 1566-1570, Dectember 1986

[10] L. Y. Chiang, J. Bhonsle, L. Wang, S.F. Shu, T.M. Chang, and J. R. Hwu, Efficient one-flask synthesis of water-soluble fullerenols, Tetrahedron, vol. 52, pp. 4963-4972, April 1996.

[11] Y. M. She, Y. P. Ji, Y. F. He, S. and Y. Liu, Detection of Specific Noncovalent Protein fullerenols Complexes by Matrix assisted Laser Desorption Ionization Mass Spectrometry, Chem Res Chin Univ, vol. 19, pp. 1735-1738, November 1998

[12] Y. F. Gao, T. Yang, X. L. Yang, Y. S. Zhang, B. L. Xiao, J. Hong, N. 
Sheibani, H. Ghourchian, T. Hong, and A. A. Moosavi-Movahedi, Direct electrochemistry of glucose oxidase and glucose biosensing on a hydroxyl fullerenes modified glassy carbon electrode, Biosensors and Bioelectronics, vol. 60, pp. 30-34, October 2014.

[13] X. L. Yang, Y. S. Zhang, T. Yang, F. Y. Geng, D. Li, B. L. Xiao, J. Hong , A. A. Moosavi-Movahedi, and H. Ghourchian, A soft-template nanostructured peroxidase based on cytochrome c and sodium decyl sulfate and its electrochemical properties on hydroxyl fullerenes modified glassy carbon electrode, Journal of the Iranian Chemical Society, vol. 13, pp. 471-479, March 2016.

[14] J. Hong, W. Wang, K. Huang, W. Y. Yang, Y. X. Zhao, B. L. Xiao, Y. F. Gao, Z. Moosavi-Movahedi, S. Ahmadian, M. Bohlooli, A. A. Saboury, H. Ghourchian, N. Sheibani, and A. A. Moosavi-Movahedi, A self-assembled nano-cluster complex based on cytochrome c and nafion: An efficient nanostructured peroxidase, Biochemical Engineering Journal, vol. 65, pp. 16-22, June 2012.

[15] J. Hong, K. Huang, W. Wang, W. Y. Yang, Y. X. Zhao, B. L. Xiao, Z. Moosavi-Movahedi, H. Ghourchian, M. Bohlooli, N. Sheibani, and A. A. Moosavi-Movahedi, Cytochrome c embraced in sodium dodecyl sulfate nano-micelle as a homogeneous nanostructured peroxidase, Journal of the Iranian Chemical Society, vol. 9, pp. 775-782, October 2012.

[16] J. Hong, K. Huang, W. Wang, W.Y. Yang, Y.X. Zhao, B.L. Xiao, Z. Moosavi-movahedi, H. Ghourchian, N. Sheibani, A.A. Moosavi-movahedi, Direct electrochemistry of artificial peroxidase based on self-assembled cytochrome c-SDS-nano-micelle. Ananlytical letters, vol. 45, pp. 2221-2235, April 2012.

[17] T. Yang, Y.S. Zhang, X.L. Yang, F.Y. Geng, B.L. Xiao, M.M. Xu, D. Li, J. Hong, and A. A. Moosavi-Movahedi, A novel self-assembled nano micelle as a highly efficient artificial peroxidase based on hexadecyl trimethyl ammonium bromide and cytochrome c, Bio-Medical Materials and Engineering, vol. 26, pp. 73-79, August 2015.

[18] Y. F. Gao, Y. X. Zhao, T. Yang, B. L. Xiao, Y. S. Zhang, X. L. Yang, J. Hong , H. Ghourchian, N. Sheibani, and A. A. Moosavi-Movahedi, A nano self-assembled artificial peroxidase: spectroscopic and electrochemical investigations, Journal of the Iranian Chemical Society, vol. 11, pp. 1397-1405, January 2014.

[19] J. Hong, W. Wang, K. Huang, W. Y. Yang, Y. X. Zhao, B. L. Xiao, Y. F. Gao, Z. Moosavi-Movahedi,H. Ghourchian, and A. A. Moosavi-Movahedi, A Highly Efficient Nano-Cluster Artificial Peroxidase and Its Direct Electrochemistry on a Nano Complex
Modified Glassy Carbon Electrode, Anal Sci, vol. 28, pp. 711-716, July 2012.

[20] J. Hong, A. A. Moosavi-Movahedi, H. Ghourchian, A. M. Rad, and S Rezaei-Zarchi, Direct electron transfer of horseradish peroxidase on Nafion-cysteine modified gold electrode, Electrochim Acta, vol. 52, pp. 6261-6267, April 2007.

[21] J. Hong, W.Y. Yang, Y.X. Zhao, B.L. Xiao, Y.F. Gao, T. Yang, H. Ghourchian, Z. Moosavi-Movahedi, N. Sheibani, J.G. Li, A. A Moosavi-Movahedi, Catalase immobilized on a functionalized multi-walled carbon nanotubes-gold nanocomposite as a highly sensitive bio-sensing system for detection of hydrogen peroxide, Electrochimica Acta, vol. 89, pp. 317-325, November 2013.

[22] E. Laviron, Adsorption, autoinhibition and autocatalysis in polarography and in linear potential sweep voltammetry, J Electroanal Chem Interfacial Electrochem, vol. 52, pp. 355-393, May 1974.

[23] H. Ma, N. Hu, and J. F. Rusling, Electroactive myoglobin films grown layer-by-layer with poly (styrenesulfonate) on pyrolytic graphite electrodes, Langmuir, vol. 16, pp. 4969-4975, April 2000.

[24] W. Y. Yang, J. Hong, Y. X. Zhao, B. L. Xiao, Y. F. Gao, T. Yang, A A Moosavi-Movahedi, H. Ghourchian, and Z. Moosavi-movahedi, Electrochemical Study of a Nano Vesicular Artificial Peroxidase on a Functional Nano Complex Modified Glassy Carbon Electrode, Journal of New Materials for Electrochemical Systems, vol. 16, pp. 89-95, April 2013.

[25] J. J. Xu, D. M. Zhou, and H. Y. Chen, A Reagentless Hydrogen Peroxide Biosensor Based on the Coimmobilization of Thionine and Horseradish Peroxidase by Their Cross-Linking with Glutaraldehyde on Glassy Carbon Electrode, Electroanalysis, vol. 10, pp. 713-716, August 1998

[26] B. Wang and S Dong, Sol-gel-derived amperometric biosensor for hydrogen peroxide based on methylene green incorporated in Nafion film, Talanta, vol. 51, pp. 565-572, March 2000.

[27] R. Yan, F. Q. Zhao, J. W. Li, F. Xiao, S. S. Fan, and B. Z. Zeng, Direct electrochemistry of horseradish peroxidase in gelatin-hydrophobic ionic liquid gel films, Electrochimica acta, vol 52, pp. 7425-7431, September 2007.

[28] Y. Xiao, H. X. Ju, and H. Y. Chen, Hydrogen peroxide sensor based on horseradish peroxidase-labeled Au colloids immobilized on gold electrode surface by cysteamine monolayer, Analytica Chimica Acta, vol. 391, pp. 73-82, May 1999. 